\title{
Magneto-transport in a mesoscopic ring with Rashba and Dresselhaus spin-orbit interactions
}

\author{
Santanu K. Maiti,, , , * Moumita Dey, ${ }^{1}$ Shreekantha Sil, ${ }^{3}$ Arunava Chakrabarti, ${ }^{4}$ and S. N. Karmakar ${ }^{1}$ \\ ${ }^{1}$ Theoretical Condensed Matter Physics Division, Saha Institute of Nuclear Physics, \\ Sector-I, Block-AF, Bidhannagar, Kolkata-700 064, India \\ ${ }^{2}$ Department of Physics, Narasinha Dutt College, 129 Belilious Road, Howrah-711 101, India \\ ${ }^{3}$ Department of Physics, Visva-Bharati, Santiniketan, West Bengal-731 235, India \\ ${ }^{4}$ Department of Physics, University of Kalyani, Kalyani, West Bengal-741 235, India
}

\begin{abstract}
Electronic transport in a one-dimensional mesoscopic ring threaded by a magnetic flux is studied in presence of Rashba and Dresselhaus spin-orbit interactions. A completely analytical technique within a tight-binding formalism unveils the spin-split bands in presence of the spin-orbit interactions and leads to a method of determining the strength of the Dresselhaus interaction. In addition to this, the persistent currents for ordered and disordered rings have been investigated numerically. It is observed that, the presence of the spin-orbit interaction, in general, leads to an enhanced amplitude of the persistent current. Numerical results corroborate the respective analytical findings.
\end{abstract}

PACS numbers: 73.23.-b, 73.23.Ra., 73.21.Hb

\section{INTRODUCTION}

The spin-orbit (SO) interaction in mesoscopic and nano-scale semiconductor structures has been at the center stage of research in condensed matter theory and device engineering in recent times. The principal reason is its potential application in spintronics, where the possibility of manipulating and controlling the spin of the electron rather than its charge, plays the all important role 1 11].

The spin-orbit fields in a solid are called the RSOI or the DSOI depending on whether the electric field originates from a structural inversion asymmetry or the bulk inversion asymmetry respectively [12]. Quantum rings formed at the interface of two semiconducting materials are ideal candidates where the interplay of the two kinds of SOI might be observed. A quantum ring in a heterojunction is realized when a two dimensional gas of electrons is trapped in a quantum well due to the band offset at the interface of two different semiconducting materials. This band offset creates an electric field which may be described by a potential gradient normal to the interface 13]. The potential at the interface is thus asymmetric, leading to the presence of a RSOI. On the other hand, at such interfaces, the bulk inversion symmetry is naturally broken.

In addition to this, the topology of such a ring gives rise to remarkable properties typical of low dimensional systems, for example, the persistent current. The phenomenon of persistent current in a conducting mesoscopic ring threaded by an Aharonov-Bohm (AB) flux $\phi$ has been established over many decades. The existence of discrete energy levels and large phase coherence length allow a non-decaying current upon the application of an

*Electronic address: santanu.maiti@saha.ac.in external magnetic flux $\phi$. Büttiker et al. [14] first studied the behavior of persistent current in a metallic ring, and then many attempts have been made to explore the basic mechanisms of persistent current in mesoscopic ring and cylindrical systems [15 22]. Later, several experiments 23 29] have also been performed to verify the existence of non-decaying current in these systems.

Although the studies involving the mesoscopic rings have already generated a wealth of literature there is still need to look deeper into the problem both from the

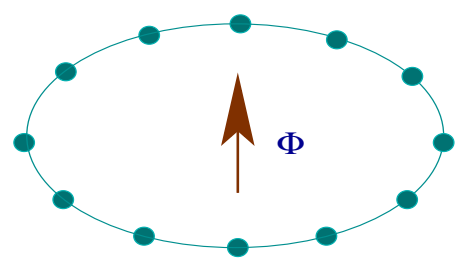

FIG. 1: (Color on-line) A mesoscopic ring threaded by an AB flux $\phi$.

point of view of fundamental physics and to resolve a few issues that have not yet been answered in an uncontroversial manner. In the present communication we undertake an in-depth analysis of the band structure of a mesoscopic ring in the presence of both the RSOI and the DSOI within a tight-binding (TB) formalism. We wish to look for a possible method of determining the strength of the DSOI in a simply connected mesoscopic ring. In recent past there have been a few experiments to measure the strength of the DSOI by optically monitoring the spin precession of the electrons [12, 30], through a measurement of the electrical conductance of narrow quantum wires defined in a 2DEG [31, 32] or, by photogalvanic methods 33. Several studies already exist which deal with electron spin states in mesoscopic rings in presence of both RSOI and DSOI and the interplay between them [9], the persistent current in presence of SO interaction [10] and the spin-Hall conductance 34] 
using the continuum as well as the tight-binding Hamiltonians. However, an analytical proposal to measure the strength of the DSOI, particularly within a tight-binding formalism, is still lacking, to the best of our knowledge.

In the present work we consider a mesoscopic ring threaded by an AB flux, and work within a tight-binding approximation. Both the RSOI and the DSOI are included in the Hamiltonian. While looking for a way to estimate the strength of the DSOI, we work out a method to calculate the energy dispersion of the mesoscopic ring either with RSOI or with the DSOI, thus gaining a clean insight into the band structure. This method leads finally to the fact that by making the strengths of the RSOI and the DSOI equal, one comes across an absolute minimum in the conductance of the ring. As the RSOI is tuned, and hence estimated by controlling an external gate voltage, the estimation of the DSOI becomes obvious. The conductance is calculated via the Drude weight 35] numerically, and is found to support the analytical understanding.

In the second part, we show that the presence of the SO interaction leads to an enhancement of persistent current in one-dimensional rings. In addition to these, we present the detailed energy band structures and the oscillations of persistent current as the RSOI is varied to get a complete picture of the phenomena at the microscopic level.

\section{THE MODEL}

Let us start by referring to Fig. 1] where a mesoscopic ring is subject to an $\mathrm{AB}$ flux $\phi$ (measured in unit of the elementary flux quantum $\left.\phi_{0}=c h / e\right)$. Within a TB framework the Hamiltonian for such an $N$-site ring is 9 , 10, 34] (and references therein),

$$
H=H_{0}+H_{s o}
$$

Here,

$$
H_{0}=\sum_{n} \boldsymbol{c}_{\boldsymbol{n}}^{\dagger} \boldsymbol{\epsilon}_{\mathbf{0}} \boldsymbol{c}_{\boldsymbol{n}}+\sum_{n}\left(\boldsymbol{c}_{\boldsymbol{n}}^{\dagger} \boldsymbol{t} e^{i \theta} \boldsymbol{c}_{\boldsymbol{n}+\mathbf{1}}+\text { h.c. }\right)
$$

and,

$$
H_{s o}=-\sum_{n}\left[\boldsymbol{c}_{n}^{\dagger} \boldsymbol{t}_{\boldsymbol{s o}} e^{i \theta} \boldsymbol{c}_{\boldsymbol{n}+\mathbf{1}}^{\dagger}+\text { h.c. }\right]
$$

where,

$$
\begin{aligned}
\boldsymbol{t}_{\boldsymbol{s o}} & =i t_{R s o}\left(\boldsymbol{\sigma}_{\boldsymbol{x}} \cos \varphi_{n, n+1}+\boldsymbol{\sigma}_{\boldsymbol{y}} \sin \varphi_{n, n+1}\right) \\
& -i t_{D s o}\left(\boldsymbol{\sigma}_{\boldsymbol{y}} \cos \varphi_{n, n+1}+\boldsymbol{\sigma}_{\boldsymbol{x}} \sin \varphi_{n, n+1}\right) .
\end{aligned}
$$

$n=1,2, \ldots, N$ is the site index along the azimuthal direction $\varphi$ of the ring. The other factors in Eqs. 2 and 4 are as follows.

$\boldsymbol{c}_{\boldsymbol{n}}=\left(\begin{array}{c}c_{n \uparrow} \\ c_{n \downarrow}\end{array}\right) ; \boldsymbol{\epsilon}_{\mathbf{0}}=\left(\begin{array}{cc}\epsilon_{0} & 0 \\ 0 & \epsilon_{0}\end{array}\right) ; \boldsymbol{t}=t\left(\begin{array}{cc}1 & 0 \\ 0 & 1\end{array}\right)$.

Here $\epsilon_{0}$ is the site energy of each atomic site of the ring. $t$ is the nearest-neighbor hopping integral and $\theta=2 \pi \phi / N$ is the phase factor due to the $\mathrm{AB}$ flux $\phi$ threaded by the ring. $t_{R s o}$ and $t_{D s o}$ are the isotropic nearest-neighbor transfer integrals which measure the strengths of Rashba and Dresselhaus SO couplings, respectively, and $\varphi_{n, n+1}=\left(\varphi_{n}+\varphi_{n+1}\right) / 2$, where $\varphi_{n}=2 \pi(n-1) / N . \quad \sigma_{\boldsymbol{x}}$ and $\boldsymbol{\sigma}_{\boldsymbol{y}}$ are the Pauli spin matrices. $c_{n \sigma}^{\dagger}\left(c_{n \sigma}\right)$ is the creation (annihilation) operator of an electron at the site $n$ with $\operatorname{spin} \sigma(\uparrow, \downarrow)$. Throughout the presentation we choose the units where $c=e=h=1$ and measure the SO coupling strength in unit of $t$.

\section{DETERMINATION OF THE DSOI}

An elementary analysis of the effect of the SO interaction on the spectral and transport properties of the Hamiltonian may now be in order. To this end, we consider a mesoscopic ring with RSOI and DSOI, but without any $\mathrm{AB}$ flux threading the ring. The SO part of the Hamiltonian is then re-written as,

$$
H_{s o}=-\sum_{n}\left[c_{n}^{\dagger} t_{s o} c_{n+1}^{\dagger}+\text { h.c. }\right]
$$

which, on expansion becomes,

$$
H_{s o}=-t \sum_{n} \boldsymbol{c}_{n}^{\dagger} \boldsymbol{c}_{\boldsymbol{n}+\mathbf{1}}-i \sum_{n} \boldsymbol{c}_{\boldsymbol{n}}^{\dagger}\left(\begin{array}{cc}
0 & \mathcal{P} \\
\mathcal{Q} & 0
\end{array}\right) \boldsymbol{c}_{\boldsymbol{n}+\mathbf{1}}+\text { h.c. }
$$

where,

$$
\begin{aligned}
\mathcal{P} & =\left(t_{\text {Rso }}+i t_{D s o}\right) \cos \varphi_{n, n+1} \\
& -i\left(t_{\text {Rso }}-i t_{D s o}\right) \sin \varphi_{n, n+1} \\
\mathcal{Q} & =\left(t_{\text {Rso }}-i t_{D s o}\right) \cos \varphi_{n, n+1} \\
& +i\left(t_{\text {Rso }}+i t_{D s o}\right) \sin \varphi_{n, n+1}
\end{aligned}
$$

A straightforward algebra helps us to recast the above Hamiltonian in Eq. 5 in the form,

$$
H_{s o}=-i \sqrt{t_{R s o}^{2}+t_{D s o}^{2}} \sum_{n} \mathcal{F}_{n, n+1} \boldsymbol{c}_{\boldsymbol{n}}^{\dagger} \boldsymbol{M} \boldsymbol{c}_{\boldsymbol{n}+\mathbf{1}}+\text { h.c. }
$$

where,

$$
\mathcal{F}_{n, n+1}=\sqrt{1-\sin (2 \xi) \sin \left(2 \varphi_{n, n+1}\right)}
$$

and,

$$
M=\left(\begin{array}{cc}
0 & e^{-i \chi_{n, n+1}} \\
e^{i \chi_{n, n+1}} & 0
\end{array}\right)
$$

with $\xi=\tan ^{-1}\left(\frac{t_{D s o}}{t_{R s o}}\right)$ and,

$\tan \chi_{n, n+1}=\frac{\sin \left(\varphi_{n, n+1}-\xi\right)}{\cos \left(\varphi_{n, n+1}+\xi\right)}$.

We now analyze Eq. 7 for two different cases. 
- Ring with RSOI only: In this limit Eq. 7 leads to the full Hamiltonian of a ring with $N$ sites. With $\epsilon_{0}=0$, and with RSOI alone, this reads,

$$
\begin{aligned}
H_{R} & =-t \sum_{n}\left(c_{n}^{\dagger} c_{n+1}+c_{n+1}^{\dagger} c_{n}\right) \\
& -i \sum_{n} c_{n}^{\dagger}\left(\begin{array}{cc}
0 & e^{-i \varphi_{n, n+1}} \\
e^{i \varphi_{n, n+1}} & 0
\end{array}\right) \boldsymbol{c}_{\boldsymbol{n}+\mathbf{1}}+\text { h.c. }
\end{aligned}
$$

To get the energy dispersion relations analytically, we define a unitary operator

$$
\mathcal{U}_{n}=\frac{1}{\sqrt{2}}\left(\begin{array}{cc}
1 & -1 \\
e^{2 \pi(n-1 / 2) i / N} & e^{2 \pi(n-1 / 2) i / N}
\end{array}\right)
$$

which transforms the old operators $\boldsymbol{c}_{\boldsymbol{n}}$ to a set of new operators $\tilde{\boldsymbol{c}_{n}}=\mathcal{U}_{n}^{\dagger} \boldsymbol{c}_{n}$. With the operators defined in the new basis, and using the discrete Fourier transform,

$$
\tilde{\boldsymbol{c}}_{\boldsymbol{n}}=\frac{1}{\sqrt{N}} \sum_{k_{m}} e^{i k_{m} a} \boldsymbol{c}_{\boldsymbol{k}}
$$

where, $a$ is the lattice spacing (and set equal to unity in the subsequent discussion), one obtains explicit expressions of eigenvalues as,

$$
\begin{aligned}
E_{k_{m},+} & =-2 t \cos (\pi / N) \cos \left(k_{m}+\frac{\pi}{N}\right) \\
& +2 \sin \left(k_{m}+\frac{\pi}{N}\right) \sqrt{t^{2} \sin ^{2}\left(\frac{\pi}{N}\right)+t_{R s o}^{2}}
\end{aligned}
$$

and,

$$
\begin{aligned}
E_{k_{m},-} & =-2 t \cos (\pi / N) \cos \left(k_{m}+\frac{\pi}{N}\right) \\
& -2 \sin \left(k_{m}+\frac{\pi}{N}\right) \sqrt{t^{2} \sin ^{2}\left(\frac{\pi}{N}\right)+t_{R s o}^{2}}
\end{aligned}
$$

where, $k_{m}=2 m \pi / N$. In presence of a magnetic flux $k_{m}$ is replaced by $\left(k_{m}+\theta\right)$.

A similar analysis can be done to extract the eigenvalues for the dispersion relations of a ring with DSOI only, that is, with RSOI set equal to zero. However, in presence of both the RSOI and DSOI one has to resort to numerical methods for calculating the eigenvalues and eigenstates.

- Analytical argument for an estimation of DSOI: The expression of $\mathcal{F}$ (Eq. 8) plays a key-role in providing a method of estimation of the strength of the DSOI. In the expression of $\mathcal{F}$, the presence of the term $\sin (2 \xi) \sin \left(2 \varphi_{n, n+1}\right)$ under the square root 'generates' an effective site-dependent hopping integral in the Hamiltonian. This site dependence of the hopping integral enters through the $\sin \left(2 \varphi_{n, n+1}\right)$ term, and is responsible for scattering as the electron circulates along the arm of the ring. The scattering reduces the conductivity (the Drude weight). From the expression of the factor $\mathcal{F}_{n, n+1}$, we see that, in the absence of $t_{D s o}$, $\xi=0$, and the the effective hopping integral becomes site-independent. The transport is ballistic in this case. On the other hand, when $t_{D s o}=t_{R s o}$ the fluctuation part under the square-root is maximum. In this case $\sin 2 \xi=1$. The site dependent scattering is strongest in this case, and should produce a minimum in the conductivity. This is precisely what we observe in the numerical calculations.

- Numerical argument for an estimation of DSOI: We now present numerical results in support of this argument, and in addition to this, on the effect of SO interaction on the persistent current in such rings. While

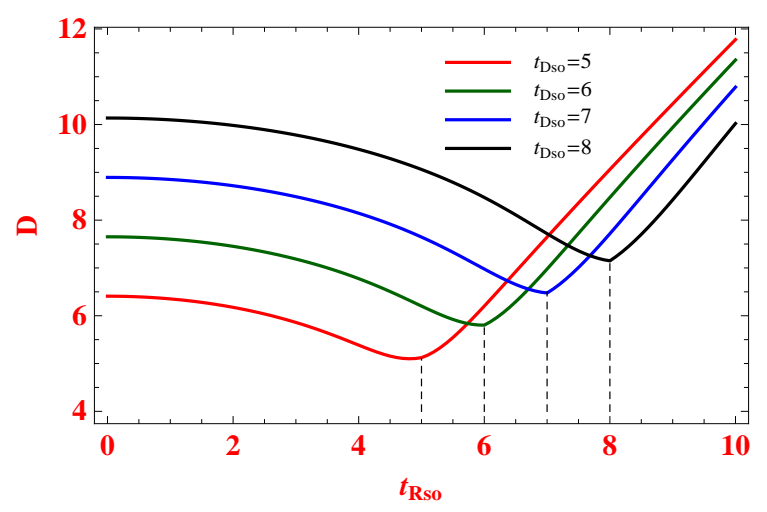

FIG. 2: (Color on-line) Drude weight $D$ (in unit of $N / 4 \pi^{2}$ ) as a function of Rashba SO coupling strength $t_{R s o}$ for a 40 -site ordered ring in the half-filled case considering different values of $t_{D s o} . D$ reaches a minimum when $t_{D s o}=t_{R s o}$.

the results in the former case completely agrees with the predictions from the analytical calculations, the latter results exhibit remarkable variations including a marked enhancement in the amplitude of the persistent current in the presence of RSOI alone.

We obtain numerical results for the conductance of a ring with different values of $t_{R s o}$ and $t_{D s o}$ by calculating the Drude weight $D$ in accordance with the idea originally put forward by Kohn [35]. The Drude weight for the ring is given by the relation,

$$
D=\left.\frac{N}{4 \pi^{2}}\left(\frac{\partial^{2} E_{0}(\phi)}{\partial \phi^{2}}\right)\right|_{\phi \rightarrow 0}
$$

where, $N$ gives total number of atomic sites in the ring. Kohn has shown that for an insulating system $D$ decays exponentially to zero, while it becomes finite for a conducting system.

We have investigated the variation of the Drude weight $D$ as a function of the strength of the RSOI for an ordered ring at half-filling. The results are depicted in Fig. 22 A remarkable feature of the results presented is that, the conductivity (Drude weight) exhibits an absolute minimum whenever the strength of the DSOI becomes equal to the strength of the RSOI. This has been verified by choosing different values of $t_{D \text { so }}$ for a 40-site ordered ring and with electron number $N_{e}=40$. In each case, the conductance minimum appears exactly at $t_{R s o}=t_{D s o}$ 

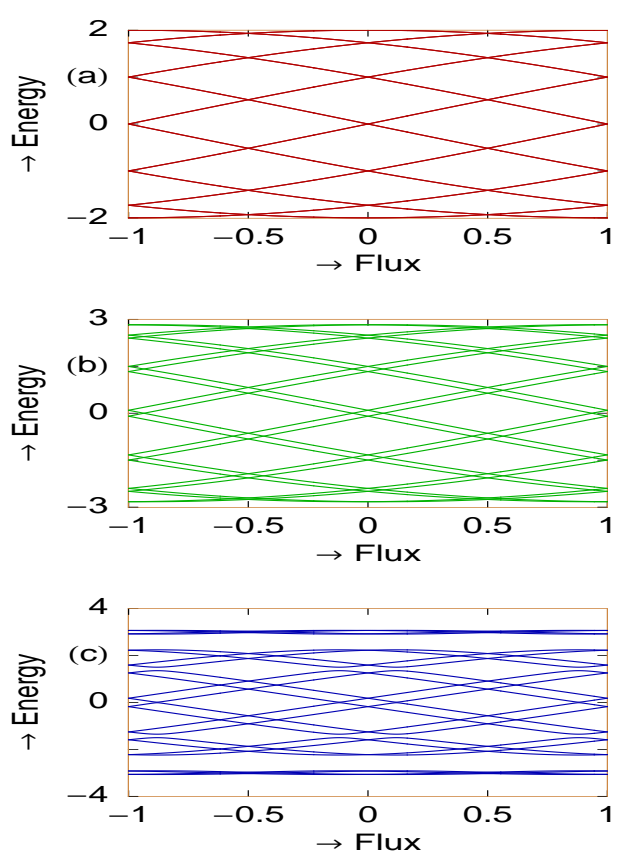
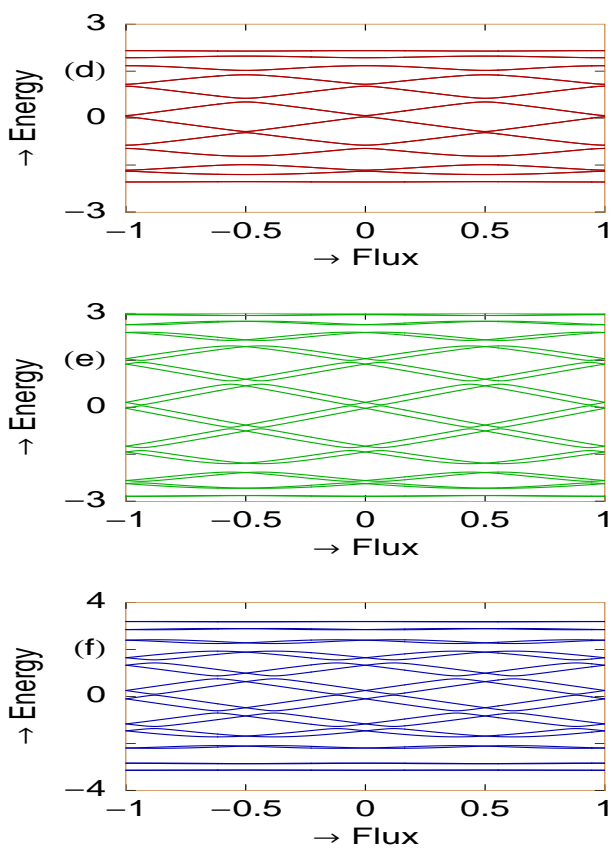

FIG. 3: (Color on-line) $E$ - $\phi$ curves of a 12-site ring, where the 1st and 2nd columns correspond to the results for the ordered $(W=0)$ and disordered $(W=1)$ cases, respectively. The red, green and blue lines correspond to $t_{R s o}=t_{D s o}=0 ; t_{R s o}=1$, $t_{D s o}=0$ and $t_{R s o}=1, t_{D s o}=0.5$, respectively.

confirming, as argued earlier, that a clear estimate of the strength of the DSOI may be obtained by noting the conductance minimum of a mesoscopic ring formed at the heterojuncion of two semiconduncting materials. As the strength of the RSOI is controlled by applying suitable gate voltage, the measurement of the DSOI becomes obvious.

Before we end this section we would like to point our that the conclusion regarding the minimum in the Drude weight remains independent of the numerical values chosen for $t_{R s o}$, and will occur whenever $t_{D s o}=t_{R s o}$. However, the question is about the detectability of this "minimum". In this regard, we would like to emphasize that observing the minimum numerically depends strongly on the size of the ring (that is, the number of atoms taken in the ring). One can bring down the minimum in the Drude weight to any desirable value of $t_{D s o}$ by appropriately increasing the size of the ring. For example, though in the paper we have presented results for a 40-site ring with the minima beginning at $t_{R s o}=5$, we have checked that the minimum can be brought down to say, $t_{R s o}=0.4$ by choosing a ring with 160 sites.

\section{ENHANCEMENT OF PERSISTENT CURRENT}

At absolute zero temperature $(T=0 \mathrm{~K})$, the persistent current in the ring described with fixed number of electrons $N_{e}$ is determined by,

$$
I(\phi)=-\frac{\partial E_{0}(\phi)}{\partial \phi}
$$

where, $E_{0}(\phi)$ is the ground state energy. We compute this quantity to understand unambiguously the role of the RSOI interaction alone on persistent current.

Before presenting the results for $I(\phi)$, to make the present communication a self contained study, we first take a look at the energy spectrum of both an ordered and a disordered ring with and without the SO interactions, as the flux through the ring is varied. In Fig. 3 the flux dependent spectra are shown for a 12-site ordered ring and a randomly disordered one (with diagonal disorder) in the left and the right columns respectively. Clearly, disorder destroys the band crossings observed in the ordered case. The presence of the RSOI and the DSOI also lifts the degeneracy and opens up gaps towards the edges of the spectrum.

- An ordered ring: In Fig. 4 we examine the effect of the RSOI on the persistent current of an ordered ring with 80 sites. The DSOI is set equal to zero. We have examined both the non-half-filled and half-filled band cases, but present results for the latter only to save space. With increasing strength of the RSOI the persistent current exhibits a trend of an increase in its amplitude. Local phase reversals take place together with the appearance of kinks in the current-flux diagrams which are however, not unexpected even without the RSOI, and are results of the band crossings observed in the spectra of such rings. 
The amplitude of the persistent current at a specific value of the magnetic flux is of course not predictable in any simple manner, and is found to be highly sensitive to

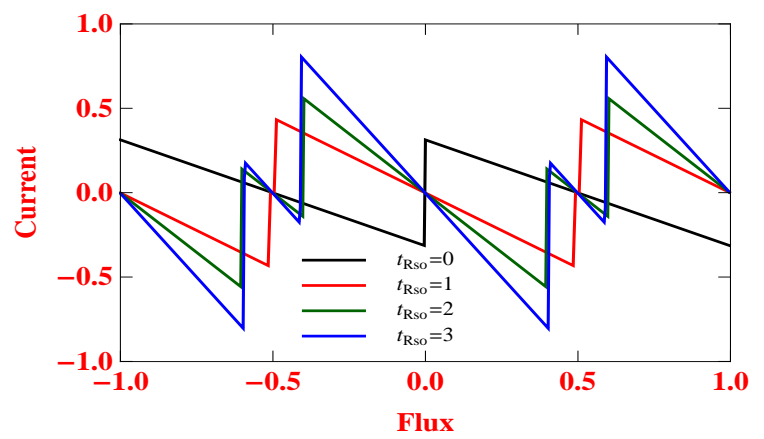

FIG. 4: (Color on-line) Current-flux characteristics of a 80site ordered $(W=0)$ half-filled ring for different values of $t_{R s o}$ when $t_{D s o}$ is set at 0 .

the number of electrons $N_{e}$ (i.e., the filling factor). Issues related to the dependence of the persistent current on the filling factor have been elaborately discussed by Splettstoesser et al. [10].

The persistent current in an ordered ring also exhibits interesting oscillations in its amplitude as the RSOI is varied keeping the magnetic flux fixed at a particular value. The oscillations persist irrespective of the band-

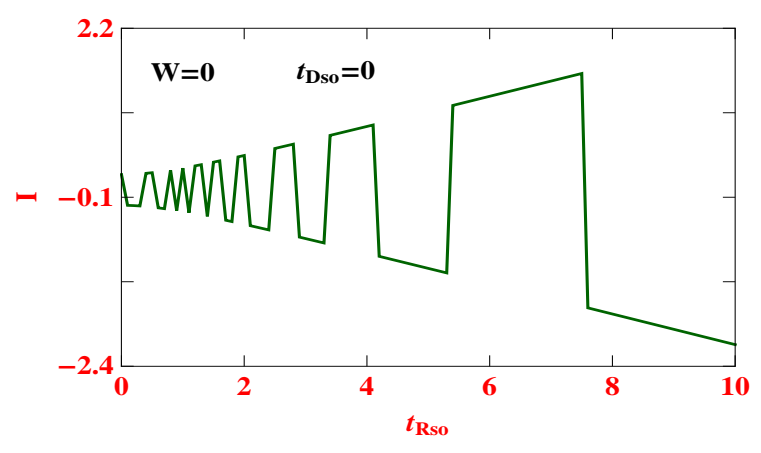

FIG. 5: (Color on-line) Persistent current at a particular AB flux $(\phi=0.25)$ as a function Rashba SO interaction strength for an ordered $(W=0)$ half-filled ring with $N=60$ when $t_{D \text { so }}$ is set to zero.

filling factor $N_{e}$, with or without the presence of the DSOI. In Fig. 5 the oscillating nature of persistent current is presented for a 60 -site ordered ring in the halffilled band case when $\phi$ is set at $\phi_{0} / 4$. The current exhibits oscillations with growing amplitude as the strength of the RSOI is increased.

- A disordered ring: We now present the results for a disordered ring of 80 sites in Fig. 6. Disorder is introduced via a random distribution (width $W=2$ ) of the values of the on-site potentials (diagonal disorder), and results averaged over sixty disorder configurations have been presented. The DSOI remains zero. Without any spin-orbit interaction, disorder completely suppresses the persistent current (an effect of the localization of the electronic states in the ring), as it is observed in Fig. 6 (black curve). With the introduction of the RSOI, the current starts increasing, and for $t_{R s o}=3$ (blue curve), increases significantly, attaining a magnitude comparable to that

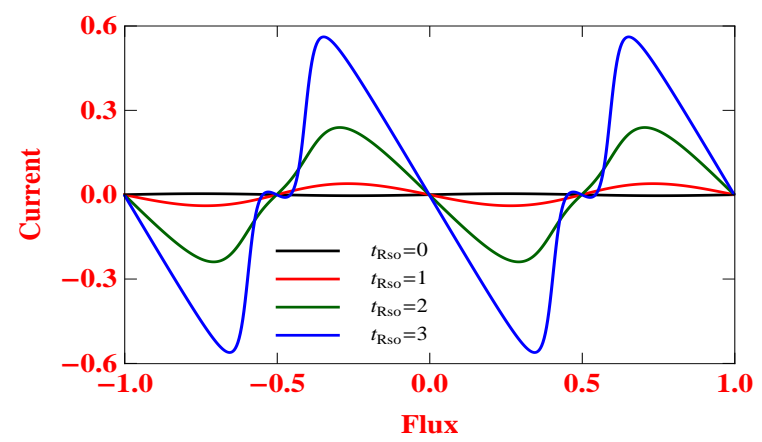

FIG. 6: (Color on-line) Current-flux characteristics of a 80site disordered ( $W=2$ ) half-filled ring for different values of $t_{R s o}$ when $t_{D s o}$ is fixed at 0 .

in a perfectly ordered ring. It is to be noted that the strength of the RSOI is strongly dependent on gate voltage. An enhancement of the persistent current in the presence of disorder can be achieved even with much lower values of the RSOI parameter compared to what have already been presented in the figures. To achieve

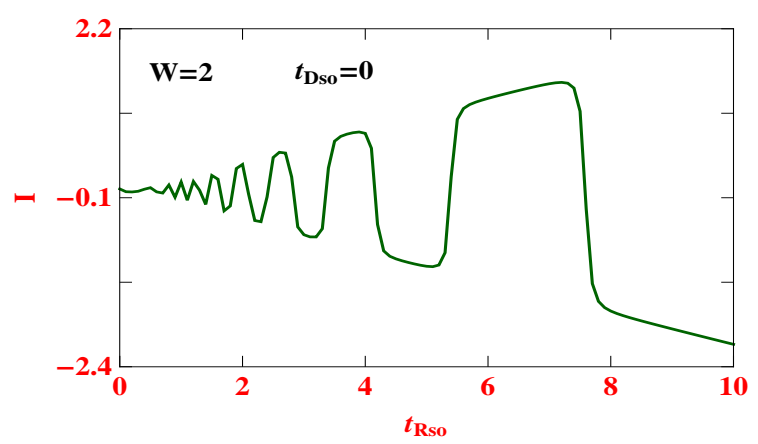

FIG. 7: (Color on-line) Persistent current at a particular AB flux $(\phi=0.25)$ as a function Rashba SO interaction strength for a disordered $(W=2)$ half-filled ring with $N=60$ when $t_{D s o}$ is set to zero.

this one needs to increase the size of the mesoscopic ring. We have checked this with a 100-site ring where even with $t_{R s o}=0.5$ the current increases by an order of magnitude compared to the case when $t_{R s o}=0$. However, we present the results using a somewhat larger values of $t_{R s o}$ for a better viewing of the results. Similar observations are made by setting $t_{R s o}=0$ and varying $t_{D s o}$.

Disorder introduces quantum interference which leads to localization of the electronic states. RSOI, on the other hand, introduces spin flip scattering in the system, which can destroy quantum interference effect, leading to a possible delocalization of the electronic states. 
This leads to an enhancement of the persistent current in the presence of disorder. The competition between the strength of disorder and the RSOI is also apparent in Fig. 7 For small values of the RSOI, the disorder dominates. As the strength of the RSOI is increased, the spin flip scattering starts dominating over the quantum interference effect, and finally the oscillations become quite similar to that in a ballistic ring. As the SO interaction is a natural interaction for a quantum ring grafted at a heterojunction, we are thus tempted to propose that the spin-orbit interaction is responsible for an enhanced persistent current in such mesoscopic disordered rings.

Before we end this section, we would like to mention that the presence of DSOI alone leads to exactly similar results as expected, since the Rashba and the Dresselhaus Hamiltonians are related by a unitary transformation. This does not change the physics. We have also computed the persistent current in the presence of both the interactions. The amplitude of the current does not increase significantly compared to the case where only one interaction is present. However, the precise magnitude of the current is sensitive to the strength of the magnetic flux threading the ring. The observation remains valid even when the strengths of the Rashba and Dresselhaus spin-orbit interactions are the same.

\section{CONCLUSION}

In conclusion, we have investigated the spectrum and the magnetic response of a tight-binding mesoscopic ring with Rashba and Dresselhaus spin-orbit interactions both analytically and numerically. Two principal results have been obtained and discussed. First, after an exact analysis of the spin dependent Hamiltonian we argue that a minimum in the conductance of the ring system when the DSOI equal the strength of the RSOI provides a method of estimating the strength of the former. Second, we present numerical results of the band structure of the ring system when it is threaded by an AB flux $\phi$. The effect of the spin-orbit terms on the energy bands are shown. This is followed by an exhaustive numerical calculation of the persistent current in ordered and disordered rings in the presence of the spin-orbit interactions. The result for the disordered ring exhibits a large persistent current, of the same order of magnitude as that in a perfectly ordered ring, in the presence of Rashba spin-orbit interaction.
[1] I. Zutic, J. Fabian, and S. Das Sarma, Rev. Mod. Phys. 76, 323 (2004).

[2] S. Datta and B. Das, Appl. Phys. Lett. 56, 665 (1990).

[3] Mesoscopic Physics and Electronics, edited by T. Ando, Y. Arakawa, K. Furuya, S. Komiyama, and H. Nakashima (Springer, Berlin, 1998).

[4] G.-H. Ding and B. Dong, Phys. Rev. B 76, 125301 (2007).

[5] S. Bellucci and P. Onorato, Phys. Rev. B 78, 235312 (2008).

[6] A. Aharony, O. E.-Wohlman, Y. Tokura, and S. Katsumoto, Phys. Rev. B 78, 125328 (2008).

[7] A. Aharony, O. E.-Wohlman, Y. Tokura, and S. Katsumoto, Physica E 42, 629 (2010).

[8] R. Citro and F. Romeo, Phys. Rev. B 75, 073306 (2007).

[9] J. S. Sheng and K. Chang, Phys. Rev. B 74, 235315 (2006).

[10] J. Splettstoesser, M. Governale, and U. Zülicke, Phys. Rev. B 68, 165341 (2003).

[11] F. E. Meijer, A. F. Morpurgo, and T. M. Klapwijk, Phys. Rev. B 66, 033107 (2002).

[12] L. Meier, G. Salis, I. Shorubalko, E. Gini, S. Schön, and K. Ensslin, Nature Physics 3, 650 (2007).

[13] J. Premper, M. Trautmann, J. Henk, and P. Bruno, Phys. Rev. B 76, 073310 (2007).

[14] M. Büttiker, Y. Imry, and R. Landauer, Phys. Lett. 96A, 365 (1983).

[15] H. F. Cheung, Y. Gefen, E. K. Riedel, and W. H. Shih, Phys. Rev. B 37, 6050 (1988).

[16] B. L. Altshuler, Y. Gefen, and Y. Imry, Phys. Rev. Lett. 66, 88 (1991).

[17] A. Schmid, Phys. Rev. Lett. 66, 80 (1991).

[18] V. Ambegaokar and U. Eckern, Phys. Rev. Lett. 65, 381 (1990).
[19] P. A. Orellana and M. Pacheco, Phys. Rev. B 71, 235330 (2005).

[20] L. K. Castelano, G.-Q. Hai, B. Partoens, and F. M. Peeters, Phys. Rev. B 78, 195315 (2008).

[21] M. Abraham and R. Berkovits, Phys. Rev. Lett. 70, 1509 (1993).

[22] G. Bouzerar, D. Poilblanc, and G. Montambaux, Phys. Rev. B 49, 8258 (1994).

[23] L. P. Levy, G. Dolan, J. Dunsmuir, and H. Bouchiat, Phys. Rev. Lett. 64, 2074 (1990).

[24] V. Chandrasekhar, R. A. Webb, M. J. Brady, M. B. Ketchen, W. J. Gallagher, and A. Kleinsasser, Phys. Rev. Lett. 67, 3578 (1991).

[25] E. M. Q. Jariwala, P. Mohanty, M. B. Ketchen, and R. A. Webb, Phys. Rev. Lett. 86, 1594 (2001).

[26] R. Deblock, R. Bel, B. Reulet, H. Bouchiat, and D. Mailly, Phys. Rev. Lett. 89, 206803 (2002).

[27] W. Rabaud, L. Saminadayar, D. Mailly, K. Hasselbach, A. Benoit, and B. Etienne, Phys. Rev. Lett. 86, 3124 (2001).

[28] D. Mailly, C. Chapelier, and A. Benoit, Phys. Rev. Lett. 70, 2020 (1993).

[29] H. Bluhm, N. C. Koshnick, J. A. Bert, M. E. Huber, and K. A. Moler, Phys. Rev. Lett. 102, 136802 (2009).

[30] M. Studer, M. P. Walser, S. Baer, H. Rusterholz, S. Schön, D. Schuh, W. Wegscheider, K. Ensslin, and G. Salis, Phys. Rev. B 82, 235320 (2010).

[31] M. Scheid, M. Kohda, Y. Kunihashi, K. Richter, and J. Nitta, Phys. Rev. Lett. 101, 266401 (2008).

[32] A. R. Dedigama, D. Jayathilaka, S. H. Gunawardana, S. Q. Murphy, M. Edirisoorya, N. Goel, T. D. Mishima, and M. B. Santos, Springer Proceedings in Physics 119, 35 (2008). 
[33] C. Yin, B. Shen, Q. Zhang, F. Xu, N. Tang, L. Cen, X. Wang, Y. Chen, and J. Yu, Appl. Phys. Lett. 97, 181904 (2010).

[34] C. P. Moca and D. C. Marinescu, J. Phys.: Condens.
Matter 18, 127 (2006).

[35] W. Kohn, Phys. Rev. 133, A171 (1964). 